\title{
On groups whose actions on finite-dimensional CAT(0) spaces have global fixed points
}

\author{
Motoko Kato
}

April 30, 2018

\begin{abstract}
We give a criterion for group elements to have fixed points with respect to a semi-simple action on a complete $\mathrm{CAT}(0)$ space of finite topological dimension. As an application, we show that Thompson's group $T$ and various generalizations of Thompson's group $V$ have global fixed points when they act semi-simply on finite-dimensional complete CAT $(0)$ spaces, while it is known that $T$ and $V$ act properly on infinite-dimensional CAT(0) cube complexes.
\end{abstract}

\section{Introduction}

A CAT(0) space is a geodesic metric space whose geodesic triangles are no fatter than triangles in the Euclidean plane with the same edge lengths. The existence of reasonable actions on CAT(0) spaces is important information for a group, related to various properties of groups.

A group $G$ is said to have Serre's property $F A$ if every action of $G$ on a simplicial tree, which is a 1-dimensional CAT(0) cube complex, has a global fixed point. If $G$ has Serre's property $F A$ then $G$ does not split as an amalgamated product or an HNN extension.

For every $k \in \mathbb{N}$, a group $G$ is said to have property $F \mathcal{A}_{k}$ if every isometric action of $G$ on a complete CAT(0) space of topological dimension $k$ has a global fixed point ([6], [18]). We say that $G$ has property $F \mathcal{A}_{k}$ for semisimple actions if every semi-simple action of $G$ on a complete CAT(0) space of topological dimension $k$ has a global fixed point. Property $\mathrm{F} \mathcal{A}_{k}$ is related to representations of groups. For example, if a group $G$ has $\mathrm{F} \mathcal{A}_{k-1}$ then $G$ is of integral $k$-representation type $([6])$.

For $n \geq 3, \mathrm{SL}_{n}(\mathbb{Z}[1 / p])$ has property $\mathrm{F} \mathcal{A}_{n-2}$ for semi-simple actions, while it does not have property $\mathrm{F} \mathcal{A}_{n-1}([6])$. For $n \geq 4$ and $k<\min \{i\lfloor n /(i+2)\rfloor \mid$ 
$2 \leq i \leq k+1\}$, Aut $\left(F_{n}\right)$ has property $\mathrm{F} \mathcal{A}_{k}([18])$. For every $g \in \mathbb{N}$, the mapping class group of a closed orientable surface of genus $g$ has property $\mathrm{F} \mathcal{A}_{g-1}$ for semi-simple actions ([1]).

We argue property $\mathrm{F} \mathcal{A}_{k}$ for semi-simple actions for Thompson's groups $T, V$ and their generalizations. $T$ and $V$ are known as the first examples of finitely presented infinite simple groups.

It is known that Thompson's groups $T, V$ and $F$ act properly on infinitedimensional CAT(0) cube complexes ([7] for $F$ and [8]). Since $F$ admits a structure of an HNN-extention, $F$ has an isometric action without a global fixed point on a tree. On the othe hand, $F, T$ and $V$ cannot act properly on finite dimensional CAT $(0)$ complexes, since they contain free abelian subgroups of infinite rank ([5]). By considering these facts, it is natural to ask whether $T$ and $V$ can act isometrically on finite-dimensional CAT(0) complexes, without global fixed points.

In this article, we give a criterion for group elements to have fixed points with respect to a semi-simle action on a complete CAT(0) space of finite topological dimension. In the following, for a group $G$ acting on a set $A$, the support of an element $g \in G$ is $\operatorname{supp}(g)=\{a \in A \mid g a \neq a\} \subset A$. The support of a subset $L$ is $\operatorname{supp}(L)=\bigcup_{l \in L} \operatorname{supp}(l) \subset A$. For a topological space $X$, $\operatorname{dim}(X)$ denotes the topological dimension of $X$. We say $X$ is $k$-dimensional if $\operatorname{dim}(X)=k$.

Theorem 1.1. Let $G$ be a group acting faithfully on a set $A$. Let $s \in G$ be an element satisfying the following conditions: there is a sequence of subgroups $\langle s\rangle=H_{0}<H_{1}<\cdots<H_{k}<H_{k+1}=G$ with elements $g_{i} \in H_{i+1}(1 \leq i \leq k)$ such that for every $1 \leq i \leq k$,

(i) every homomorphism from $H_{i}$ to $\mathbb{R}$ is trivial, and

(ii) $g_{i}\left(\operatorname{supp}\left(H_{i}\right)\right) \cap \operatorname{supp}\left(H_{i}\right)=\emptyset$ in $A$.

Then for every complete $k$-dimensional $C A T(0)$ space $X$, for every semisimple action of $G$ on $X, s$ has a fixed point.

By using Theorem 1.1 and the following theorem by Bridson, we may confirm that many Thompson-type groups have property $\mathrm{F} \mathcal{A}_{k}$ for semi-simple actions for every $k \in \mathbb{N}$.

Theorem $1.2\left([1,[18])\right.$. Let $k_{1}, \ldots, k_{r}$ be positive integers and let $X$ be a complete $C A T(0)$ space of $\operatorname{dim}(X)<k_{1}+\cdots+k_{r}$. Let $S_{1}, \ldots, S_{r} \subset \operatorname{Isom}(X)$ be subsets with $\left[s_{i}, s_{j}\right]=1$ for all $s_{i} \in S_{i}$ and $s_{j} \in S_{j}(i \neq j)$. If each $k_{i}$ element subset of $S_{i}$ has a fixed point in $X$ for $i=1, \ldots, r$, then for some $i$, every finite subset of $S_{i}$ has a fixed point. 
Corollary 1.3. Thompson's group $T$ and $V$ have property $\mathrm{F} \mathcal{A}_{k}$ for semisimple actions for every $k \in \mathbb{N}$.

Similar arguments show that Brin-Higman-Thompson groups $n V_{q}$ and Nekrashevych-Röver groups $V_{q}(G)$ have property $\mathrm{F} \mathcal{A}_{k}$ for semi-simple actions for every $k \in \mathbb{N}$.

$T, V$ and their generalizations are known to have property FA ([8] for $T$ and $V$, [14] for $n V$ ). It is also known that any isometric action of $V$ on a finite-dimensional CAT(0) cube complex fixes a point ([12]). Corollary [1.3 generalizes these results.

Corollary 1.3 partially answers to a question in [12] (Question 1.5), which asks whether $T$ is hyperbolically elementary and whether every isometric action of $T$ on a $k$-dimensional $\mathrm{CAT}(0)$ cube complex has a global fixed point, for every $k \geq 0$.

I would like to thank Takuya Sakasai, Javier Aramayona and Yash Lodha for helpful discussions and comments. I would like to thank Anthony Genevois who let me know about results in [12] and gave many comments on this paper. This work was supported by JSPS KAKENHI Grant Number 17J07711 and the Program for Leading Graduate Schools, MEXT, Japan.

\section{Isometries of $\mathrm{CAT}(0)$ spaces and a fixed point criterion}

In this section, we compile some basic facts on isometries of complete CAT(0) spaces, mainly from [2], and then proceed to prove Theorem 1.1.

Let $(X, d)$ be a metric space and let $\gamma$ be an isometry of $X$. The translation length of $\gamma$ is the number $|\gamma|=\inf \{d(x, \gamma(x)) \mid x \in X\}$. We write $\operatorname{Min}(\gamma)=\{x \in X|d(x, \gamma(x))=| \gamma \mid\}$.

Lemma 2.1 (Proposition 6.2 of [2]). Let $X$ be a CAT(0) space and $\gamma$ be an isometry of $X . \operatorname{Min}(\gamma)$ is a closed convex subset of $X$.

An isometry $\gamma$ is called semi-simple if $\operatorname{Min}(\gamma)$ is non-empty. A semisimple isometry $\gamma$ is called hyperbolic if $|\gamma|>0$, and called elliptic if $|\gamma|=0$, i.e. $\gamma$ has a fixed point. We say that an isometric action of a group $G$ on a metric space is semi-simple if every $g \in G$ is either elliptic or hyperbolic.

The following lemma describes the structure of $\operatorname{Min}(\gamma)$ for hyperbolic isometries.

Lemma 2.2. Let $X$ be a $C A T(0)$ space and $\gamma$ be a hyperbolic isometry of $X$. 
(1) $\operatorname{Min}(\gamma)$ is isometric to a product $Y \times \mathbb{R}$, where $Y=Y \times\{0\}$ is a closed convex subspace of $X$. If $\operatorname{dim}(X)<\infty$, then $\operatorname{dim}(Y)<\operatorname{dim}(X)$.

(2) Every isometry $\alpha$ that commutes with $\gamma$ leaves $\operatorname{Min}(\gamma)=Y \times \mathbb{R}$ invariant, and its restriction to $Y \times \mathbb{R}$ is of the form $\left(\alpha_{1}, \alpha_{2}\right)$, where $\alpha_{1}$ is an isometry of $Y$ and $\alpha_{2}$ is a translation on $\mathbb{R}$.

Proof. (1) The proof of the existence of the product decomposition is in [2] (Theorem 2.14 and Theorem 6.8). We observe that $Y$ has smaller dimension than $X$. By a general fact on the topological dimension $([15]), \operatorname{dim}(Y \times \mathbb{R})=$ $\operatorname{dim}(Y)+\operatorname{dim}(\mathbb{R})=\operatorname{dim}(Y)+1$. Since $Y \times \mathbb{R}$ is a closed subspace of $X$, $\operatorname{dim}(Y \times \mathbb{R}) \leq \operatorname{dim}(X)$. It follows that $\operatorname{dim}(Y)<\operatorname{dim}(X)$.

(2) The proof is in [2] (Theorem 6.8).

Lemma 2.3 (Propositions 6.2 and 6.9 of [2]). Let $X$ be a CAT(0) space and $\gamma$ be a hyperbolic isometry of $X$.

(1) If $C \subset X$ is non-empty, complete, convex and $\gamma$-invariant, then $\gamma$ is semi-simple if and only if $\left.\gamma\right|_{C}$ is semi-simple.

(2) If $X$ splits as a product $X^{\prime} \times X^{\prime \prime}$, for every isometry $\gamma=\left(\gamma^{\prime}, \gamma^{\prime \prime}\right)$ preserving the decomposition, $\gamma$ is semi-simple if and only if $\gamma^{\prime}$ and $\gamma^{\prime \prime}$ are semi-simple.

Proof of Theorem 1.1. The proof proceeds by induction on $k$. When $k=0$, the lemma is trivial. We assume that the lemma holds for all $l<k$.

We fix a complete $\mathrm{CAT}(0)$ space $X$ of $\operatorname{dim}(X)=k$ and a semi-simple action of $G$ on $X$. Assume to the contrary that $s$ is not elliptic. By the semi-simplicity of the action, $s$ is a hyperbolic element.

By the assumption, there are $H_{k}<G$ and $g_{k} \in G$ such that $g_{k}\left(\operatorname{supp}\left(H_{k}\right)\right)$ and $\operatorname{supp}\left(H_{k}\right)$ are disjoint. Let $H_{k}^{g_{k}}=\left\{g_{k} h g_{k}^{-1} \mid h \in H_{k}\right\}$. Since $\operatorname{supp}\left(H_{k}^{g_{k}}\right)=$ $g_{k}\left(\operatorname{supp}\left(H_{k}\right)\right)$ and the action of $G$ on $A$ is faithful, $H_{k}^{g_{k}}$ and $H_{k}$ commute in $G$. By Lemma 2.2, $H_{k}^{g_{k}}$ preserves $\operatorname{Min}(s)=Y \times \mathbb{R}$, and the action of $H_{k}^{g_{k}}$ on $\operatorname{Min}(s)$ splits into two actions: an isometric action on $Y$ and an action on $\mathbb{R}$ by translations.

We first observe the action of $H_{k}^{g_{k}}$ on $\mathbb{R}$ by translations. Since every homomorphism of $H_{k}$ on $\mathbb{R}$ is trivial, $H_{k}^{g_{k}}$ fixes every point in $\mathbb{R}$.

We next observe the action of $H_{k}^{g_{k}}$ on $Y$. The action is semi-simple by Lemma 2.3. Since $Y \times\{0\}$ is a closed convex subspace of $X, Y$ is a complete CAT(0) space. By Lemma 2.2 (1), the topological dimension of $Y$ is less than $k$. By the inductive assumption to the action of $H_{k}^{g_{k}}$ on $Y, s^{g_{k}}$ fixes a point in $Y$.

It follows that $s^{g_{k}}$ fixes a point in $X$, which contradicts to the assumption that $s$ is not elliptic. 


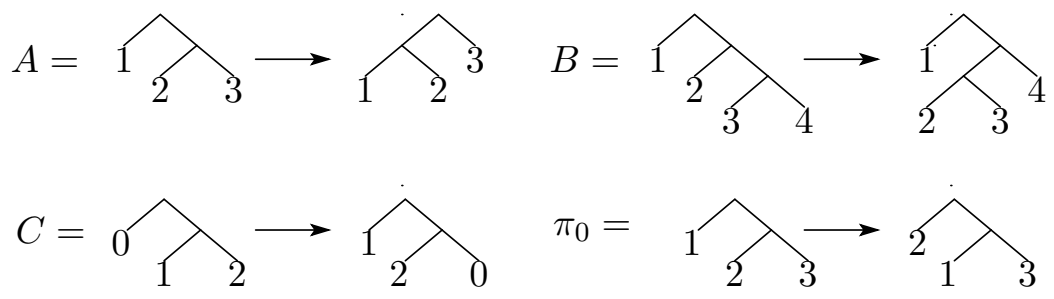

Figure 1: Generators of Thompson's groups

\section{Applications for Thompson-type groups}

\subsection{Thompson's groups $F, T$ and $V$}

Classically, there are three types of Thompson's groups: $F, T$ and $V$. We give definitions of these groups, based on [5]. A standard dyadic interval is an interval of the form $\left[a / 2^{b},(a+1) / 2^{b}\right]$ in the unit interval $[0,1]$, where $a \in \mathbb{Z}$ and $b \in \mathbb{N}$. A dyadic rational is a rational number of the form $a / 2^{b}$ in $[0,1]$.

Thompson's group $F$ is a group of piecewise linear homeomorphisms of $[0,1]$, differentiable with derivatives of powers of 2 on finitely many standard dyadic intervals.

Thompson's group $T$ is a group of piecewise linear homeomorphisms of $S^{1}$, differentiable with derivatives of powers of 2 on finitely many standard dyadic intervals. We regard $S^{1}$ as the unit interval with identified endpoints.

Thompson's group $V$ is a group of piecewise linear right-continuous bijections from $[0,1)$ to itself, differentiable with derivatives of powers of 2 on finitely many standard dyadic intervals.

We identify divisions of $[0,1]$ into finitely many standard dyadic intervals with finite rooted binary trees. Under this identification, we represent elements of Thompson's groups by a pair of finite rooted binary trees with the same number of leaves. The following figure shows generators of Thompson's groups, represented as tree pairs. $F$ is generated by $A$ and $B$ of Figure 1, $T$ is generated by $A, B$ and $C$. $V$ is generated by $A, B, C$ and $\pi_{0}$.

Remark 3.1. (cf. [5]) The commutator subgroup $[F, F]$ of $F$ is simple, and is equal to the subgroup consisting of elements which restrict to the identity on some neighborhoods of 0 and 1.

Let $G$ be $F$ or $T$. For every standard dyadic interval I properly contained in $[0,1]$, let $G^{I}=\{g \in G \mid \operatorname{supp}(g) \subset I\}<G$. Considering the canonical identification of I with $[0,1]$, we may observe that every $G^{I}$ is isomorphic to $F$. Its commutator subgroup $\left[G^{I}, G^{I}\right]$ coincides with the subgroup consisting of 
elements which restrict to the identity on some neighborhoods of two boundary points of I. Similarly, we may observe that $V^{I}$ is isomorphic to $V$, which is simple.

Lemma 3.1. (1) Let $G$ be either $T$ or $V$. Let $x$ be a dyadic rational in $S^{1}=[0,1] /\{0=1\}$. For every nonempty open subset $U_{1}$ of $S^{1}$ and every compact subset $U_{2}$ of $S^{1}-\{x\}$, there is $g \in G$ such that $g\left(U_{2}\right)$ is contained in $U_{1}$.

(2) Let $G$ be either $F, T$ or $V$. Let $I$ be a a standard dyadic interval in $[0,1]$. For every nonempty open subset $U_{1}$ of $I$ and every compact subset $U_{2}$ in the interior of $I$, there is $g$ in the commutator subgroup of $H^{I}=\{h \in G \mid \operatorname{supp}(h) \subset I\}$ such that $g\left(U_{2}\right)$ is contained in $U_{1}$.

Proof. (1) Since $V$ contains $T$ as its subset, it is enough to show (1) for $G=$ T. $U_{2}$ is included in the union of finitely many standard dyadic intervals. It is immediate from definitions that there is $g \in T$ which maps these standard dyadic intervals into a standard dyadic interval included in $U_{1}$.

(2) There is a closed interval $J$ in the interior of $I$, containing $U_{2}$. It is immediate from definitions that there is $g \in H^{J}=\{h \in G \mid \operatorname{supp}(h) \subset J\}$, satisfying required conditions. When $G=F$ or $T$, the commutator subgroup $\left[H^{I}, H^{I}\right]$ coincides with the subgroup consisting of elements which restrict to the identity on some neighborhoods of two boundary points of $I$, according to Remark 3.1. When $G=V,\left[H^{I}, H^{I}\right]=H^{I}$. In both cases, $H^{J} \subset\left[H^{I}, H^{I}\right]$ and thus $g$ is included in $\left[H^{I}, H^{I}\right]$.

For $\varepsilon>0$, we say that a subset $U$ of $[0,1]$ is of size less than $\varepsilon$ if $U$ is in the union of finitely many standard dyadic intervals whose sum of lengths is less than $\varepsilon$.

Lemma 3.2 (cf. [3]). For every $\varepsilon>0, V$ and $T$ are generated by elements with supports of size less than $\varepsilon$.

Proof. The proof for $V$ is in [3]. We show the lemma for $T$.

We take three affine copies of $[0,1]: U_{1}=[0,1 / 2], U_{2}=[1 / 4,3 / 4]$ and $U_{3}=[1 / 2,1]$. For every $i=1,2,3, F_{i}=\left\{f \in F \mid \operatorname{supp}(f) \subset U_{i}\right\}$ is isomorphic to $F$. F is generated by $F_{1}, F_{2}$ and $F_{3}$ (cf. Figure 1 and Figure 2).

We let $U_{4}=[3 / 4,1] \cup[1,1 / 4] . \quad U_{4}$ is an affine copy of $[0,1]$ in $S^{1}=$ $[0,1] /\{0=1\}$. Let $T_{i}=\left\{t \in T \mid \operatorname{supp}(t) \subset U_{i}\right\}$ for $1 \leq i \leq 4$, all isomorphic to $F$. $T_{i}=F_{i}$ for $i=1,2,3$. $T$ is generated by $T_{1}, T_{2}, T_{3}$ and $T_{4}$ (cf. Figure 1 , Figure 2 and Figure 31).

By the above observations, we may represent every element of $T$ as a composition of elements in $\bigcup_{i=1,2,3,4} T_{i}$. We may represent every element of 


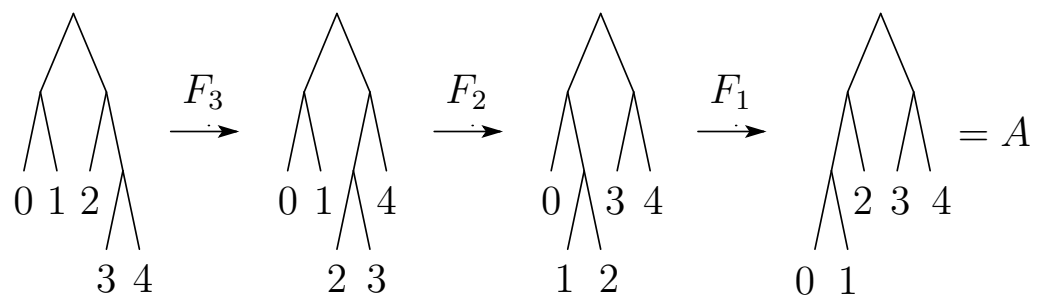

Figure 2: $A \in\left\langle F_{1}, F_{2}, F_{3}\right\rangle$
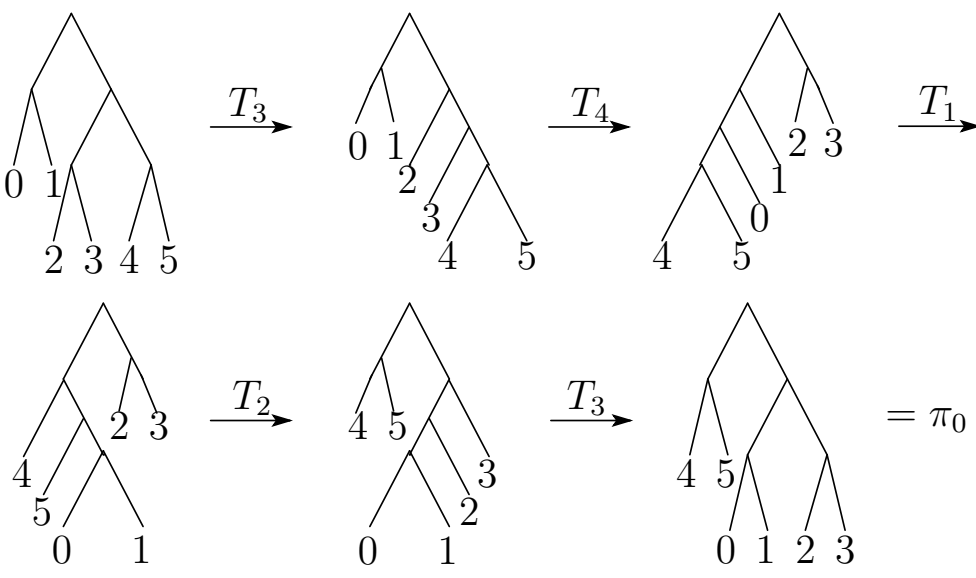

Figure 3: $C \in\left\langle T_{1}, T_{2}, T_{3}, T_{4}\right\rangle$

$T_{i}$ as a composition of elements in the copy of $\bigcup_{i=1,2,3} F_{i}$ in $T_{i} \cong F$. Repeating this process, we may represent $g$ as a composition of elements with size less than $\varepsilon$.

Proof of Corollary 1.3. Let $G$ be either $T$ or $V$. Let $k \in \mathbb{N}$. We assume that $G$ is acting semi-simply on a $k$-dimensional complete $\operatorname{CAT}(0)$ space. According to Lemma 3.2, we take a finite generating set $S$ of $G$, consisting of elements with supports of size less than $1 /(k+1)$.

We fix a sequence of standard dyadic intervals $\left\{I_{i}\right\}_{0 \leq i \leq k+1}$, where $I_{k+1}=$ $[0,1]$ and $I_{i} \subset \operatorname{Int}\left(I_{i+1}\right)$ for every $0 \leq i \leq k$. For every $s \in S$, there is a dyadic rational $x \in[0,1]-\operatorname{supp}(s)$. We apply Lemma 3.1 (1) to the interior of $I_{0}$ and a proper compact subset in $[0,1]-\{x\}$ including $\operatorname{supp}(s)$, and take $g_{0} \in G$ such that $\operatorname{supp}\left(s^{g_{0}}\right)=g_{0}(\operatorname{supp}(s)) \subset \operatorname{Int}\left(I_{0}\right)$.

For every $1 \leq i \leq k$, we let $H_{i}$ be the commutator subgroup of $H^{I_{i}}=$ $\left\{h \in G \mid \operatorname{supp}(h) \subset I_{i}\right\}$, which is isomorphic to either $[F, F]$ or $V$. Since both $[F, F]$ and $V$ are simple, $\left\{H_{i}\right\}_{1 \leq i \leq k}$ satisfies the condition $(i)$ in Theorem 1.1. 
For every $0 \leq i \leq k$, there is a nonempty open subset $\bar{I}_{i}$ in $I_{i+1}$ such that $I_{i} \cap \bar{I}_{i}=\emptyset$. For every $1 \leq i \leq k$, we apply Lemma 3.1 (2) to $I=I_{i+1}, U_{1}=\bar{I}_{i}$ and $U_{2}=I_{i}$, and we get $g_{i} \in H_{i+1}$ such that $g_{i}\left(I_{i}\right) \subset \bar{I}_{i} .\left\{H_{i}\right\}_{1 \leq i \leq k}$ and $\left\{g_{i}\right\}_{1 \leq i \leq k}$ satisfies the condition (ii) in Theorem 1.1. Applying Theorem 1.1 to a conjugate, every $s \in S$ is elliptic.

We fix mutually disjoint nonempty open subsets $J_{1}, \ldots, J_{k+1}$ of $[0,1]$. For every subset $S_{k+1} \subset S$ of $(k+1)$ elements, there is a dyadic rational which is not included in $\operatorname{supp}\left(S_{k}\right)$. By Lemma $3.1(1)$, there are $f_{1}, \ldots, f_{k+1} \in G$ such that $f_{i}\left(\operatorname{supp}\left(S_{k+1}\right)\right) \subset J_{i}$. By applying Theorem 1.2 to $S_{k+1}$, we see elements in $S_{k+1}$ have a common fixed point. By applying Theorem 1.2 again to $S$, we see elements in $S$ have a common fixed point, and thus there is a global fixed point for the action of $G$ on $X$.

We note that various generalizations of $V$ have property $\mathrm{F} \mathcal{A}_{k}$. For each $q \in \mathbb{N}$ where $q \geq 2$, we consider standard $q$-adic intervals, instead of standard dyadic intervals, and define Higman-Thompson groups $V_{q}$ ([13]). By definition, $V_{2}$ coincides with $V$. For each $q, n \in \mathbb{N}$ where $q \geq 2$, we consider products of $n$ standard $q$-adic intervals contained in $[0,1]^{n}$, instead of standard dyadic intervals in $[0,1]$, and define Brin-Higman-Thompson groups $n V_{q}$ ([3]). By definition, $1 V_{q}$ coincides with $V_{q}$.

There is a version of Lemma 3.2 for $n V_{q}$ (Proposition 3.2 of [3]), and we can take a generating set of $n V_{q}$, consisting of elements with supports of arbitrary small size. Since $n V_{q}$ is known to be finitely generated, we can assume that such generating set is finite. Let $I$ be the product of $n$ standard $q$-adic intervals. Lemma 3.1 holds true for $G=n V_{q}$ and $I$. If we define $\left(n V_{q}\right)^{I}$ similarly as in Remark 3.1, we may observe that $\left(n V_{q}\right)^{I}$ is isomorphic to $n V_{q}$, whose commutator subgroup is simple. These observations enables us to carry on the same arguments as in the proof of Corollary 1.3, and we get the following.

Corollary 3.3. For every $q, n \in \mathbb{N}(q \geq 2)$, $n V_{q}$ has property $\mathrm{F} \mathcal{A}_{k}$ for every $k \in \mathbb{N}$.

Another generalization of $V$ is Nekrashevych-Röver groups $V_{q}(G)$ ([10], [11], [16], [17]). We fix $q \in \mathbb{N}(q \geq 2)$ and a subgroup $G$ of the symmetric group $\mathfrak{S}_{q}$. Let $A_{q}$ be a set of alphabets $A_{q}=\{0,1, \ldots, q-1\}$. We consider an action of $G$ on $C_{q}=A_{q}^{\mathbb{N}}$, which is induced by the action of $G$ on $A_{q}$. Let $C_{q}$ be the set of all infinite words in $A_{q}$. For a finite word $w$ in $A_{q}$, let $I(w)$ be a subset of $C_{q}$, consisting of all words which start with $w$. A division of $C_{q}$ is a sequence of finite words $\left(w_{i}\right)_{1 \leq i \leq m}$ such that $\left\{I\left(w_{i}\right)\right\}_{1 \leq i \leq m}$ are mutually disjoint and cover $C_{q}$. Given a pair of divisions $W=\left(w_{i}\right)_{1 \leq i \leq m}, W^{\prime}=$ $\left(w_{i}^{\prime}\right)_{1 \leq i \leq m}$ with the same cardinality and a sequence $\left(\sigma_{i}\right)_{1 \leq i \leq m}$ of elements of 
$G$, we define a bijection $v=v\left(W, W^{\prime},\left(\sigma_{i}\right)_{i}\right)$ between $C_{q}$ by $v\left(w_{i} u\right)=w_{i}^{\prime} \sigma_{i}(u)$, for every $i$ and every suffix $u$. $V_{q}(G)$ is a group consisting of all bijections of the form $v\left(W, W^{\prime},\left(\sigma_{i}\right)_{i}\right)$.

It is known that $V_{q}(G)$ is finitely generated and virtually simple ([10]). By identifying $C_{q}$ with the unit interval and identifying subsets of the form $I(w)$ with standard $q$-adic intervals, we may observe that $V_{q}(\{1\})$ coincides with $V_{q}$. Under this identification, we may carry on the same arguments as in the proof of Corollary 1.3, and we get the following.

Corollary 3.4. For every $q \in \mathbb{N}$ and every $G<\mathfrak{S}_{n}$, Nekrashevych-Röver groups $V_{q}(G)$ has property $\mathrm{F} \mathcal{A}_{k}$ for every $k \in \mathbb{N}$.

We note that every $V_{q}(G)$ acts properly on an infinite-dimensional CAT(0) cube complex $([11])$.

\section{References}

[1] M. R. Bridson, Semisimple actions of mapping class groups on CAT(0) spaces, preprint, arXiv:0908.0685.

[2] M. R. Bridson and A. Haefliger, Metric spaces of nonpositive curvature, Grundlehren der Math. Wiss. 319, Springer-Verlag, Berlin, 1999.

[3] M. G. Brin, Higher dimensional Thompson groups, Geom. Dedicata, 108, 163-192, 2004.

[4] K. S. Brown, Finiteness properties of groups, J. Pure Appl. Algebra, 44 (1-3), 45-75, 1987.

[5] J. W. Cannon, W. J. Floyd, and W. R. Parry, Introductory notes on Richard Thompson's groups, Enseign. Math. (2) 42, 215-256, 1996.

[6] B. Farb, Group actions and Helly's theorem, Advances in Mathematics 222 1574-1588, 2009.

[7] D. S. Farley, Finiteness and CAT(0) properties of diagram groups, Topology 42 1065-1082, 2003.

[8] D. S. Farley, Actions of picture groups on CAT(0) cubical complexes, Geom. Dedicata 110, 221-242, 2005.

[9] D. S. Farley, A proof that Thompson's groups have infnitely many relative ends, J. Group Theory 14, 649-656, 2011. 
[10] D. S. Farley and B. Hughes, Finiteness properties of some groups of local similarities, arXiv:1206.2692.

[11] B. Hughes, Local similarities and the Haagerup property, with an appendix by D. S. Farley, Groups Geom. Dyn., 3, 299-315, 2009.

[12] A. Genevois, Hyperbolic and cubical rigidities of Thompson's group V, preprint, arXiv:1804.01791.

[13] G. Higman, Finitely presented infinite simple groups, Notes Pure Math. 8, Australian National University, Canberra, vii+82 pp, 1974.

[14] M. Kato, Higher dimensional Thompson groups have Serre's property FA, preprint, arXiv:math/1504.06680.

[15] K. Morita, Čech cohomology and covering dimension for topological spaces, Fund. Math. 87, 31-51, 1975.

[16] V. V. Nekrashevych, Cuntz-Pimsner algebras of group actions, J. Operator Theory, 52 (2), 223-249, 2004.

[17] C. E. Röver, Constructing finitely presented simple groups that contain Grigorchuk groups, J. Algebra, 220 (1), 284-313, 1999.

[18] O. Varghese, Fixed points for actions of Aut(Fn) on CAT(0) spaces, Münster J. of Math. 7, 439-462, 2014. 